\title{
Direct Compression by Megadolichobasilar Anomaly as a Cause of Trigeminal Neuralgia; A Case Diagnosed by MRI
}

\author{
Motoaki Yoshida and Michiko Asano* \\ Department of Visually Impaired, Tsukuba College of \\ Technology, Tsukuba 305, and *Institute of Clinical \\ Medicine, University of Tsukuba, Tsukuba 305
}

\begin{abstract}
Yoshida, M. and Asano, M. Direct Compression by Megadolichobasilar Anomaly as a Cause of Trigeminal Neuralgia; $A$ Case Diagnosed by MRI. Tohoku J. Exp. Med., 1994, $172(4), 327-332$ _ A 49 year-old man with trigeminal neuralgia caused by megadolichobasilar anomaly was described. The patient having neuralgic attacks in the second branch of the right trigeminal nerve for two and a half years showed the angiographic evidence of a tortuous and elongated vertebrobasilar artery and MRI of the brain clearly visualized the root entry zone of the nerve having been compressed by the main trunk of ectatic basilar artery. Cases of trigeminal neuralgia due to the compression by the basilar artery itself have been rarely described previously. In order to evaluate trigeminal neuralgia, MRI is essential to determine the relationship of the involved site of nerve to the pathogenic artery.— trigeminal neuralgia; megadolichobasilar anomaly; magnetic resonance imaging
\end{abstract}

Vascular compression is one of the major pathogenic factors in trigeminal neuralgia. Surgical neurovascular decompression is used as a treatment of choice. In general, vessels involved are rather small ones such as the superior cerebellar artery, anterior inferior cerebellar artery and petrosal vein. On the other hand, trigeminal neuralgia caused by an ectatic basilar artery itself has been rarely described; e.g., Janneta reported no such cases in his series of 60 cases with trigeminal neuralgia due to vascular compression (Janneta 1977).

It is a prerequisite for surgeons to be acquainted precisely with neurovascular relationships resposible for trigeminal neuralgia prior to surgical intervention. We report here a case with trigeminal neuralgia caused by a megadolichobasilar anomaly and the usefulness of MRI to reveal the site of nerve compression.

\section{CASE Report}

A 49 year-old man with two and a half year history of trigeminal neuralgia was

Received October 6, 1992; revision accepted for publication March 3, 1994.

Address for reprints: Motoaki Yoshida, M.D., Department of Visually Impaired, Tsukuba College of Technology, Tsukuba 305, Japan. 
admitted to Tsukuba University Hospital for the investigation of a prepontine abnormal shadow revealed by CT. Sharp pain was precipitated in the right 2nd division of trigeminal nerve, when eating or washing his face. The pain was precipitated, even when salivation was induced by eating sour foods such as lemons or sometimes only by looking at those foods. The trigger zone was located at the right cheek just lateral to his nose. He had been treated effectively with $30 \mathrm{mg}$ of baclofen and $200 \mathrm{mg}$ of carbamazepine as an outpatient. At the age of 34 , he had received surgical treatment for hydronephrosis caused by renal stone. Since 3 years ago, he has been on thiazide for hypertension. He was obese and blood pressure was $150 / 90 \mathrm{mmHg}$. There was mild Horner syndrome at the right side. Corneal reflex was normal. There was neither sensory impairement in the face nor weakness in masseter muscles. Other neurological examinations revealed no abnormalities. Urinalysis and hemogram were normal. Serum uric acid was $8.1 \mathrm{mg} / 100 \mathrm{ml}$ (NR 3.5-7.8), triglyceride $254 \mathrm{mg} / 100 \mathrm{ml}$ (NR 40-170) and total cholesterol $270 \mathrm{mg} / 100 \mathrm{ml}$ (NR 120-220). Fasting blood sugar, urea-N and creatinine were normal. ECG showed left ventricular high voltage and large negative $T$ in $\mathrm{V}_{2}-\mathrm{V}_{6}$.

Brain MRI revealed the main trunk of ectatic basilar artery compressing the root entry zone of the right trigeminal nerve and indenting the lateral aspect of pons (Fig. 1).

Cerebral angiography revealed an ectatic, tortuous vertebrobasilar artery which was laterally deviated $23 \mathrm{~mm}$ from the midline at V4 portion and projected $26 \mathrm{~mm}$ above the plane of anterior cranial fossa through the anterior clinoid process at its bifurcation (Resta et al. 1984) (Fig. 2).

Since his pain was well-controlled by the medications, he was discharged without any surgical interventions.

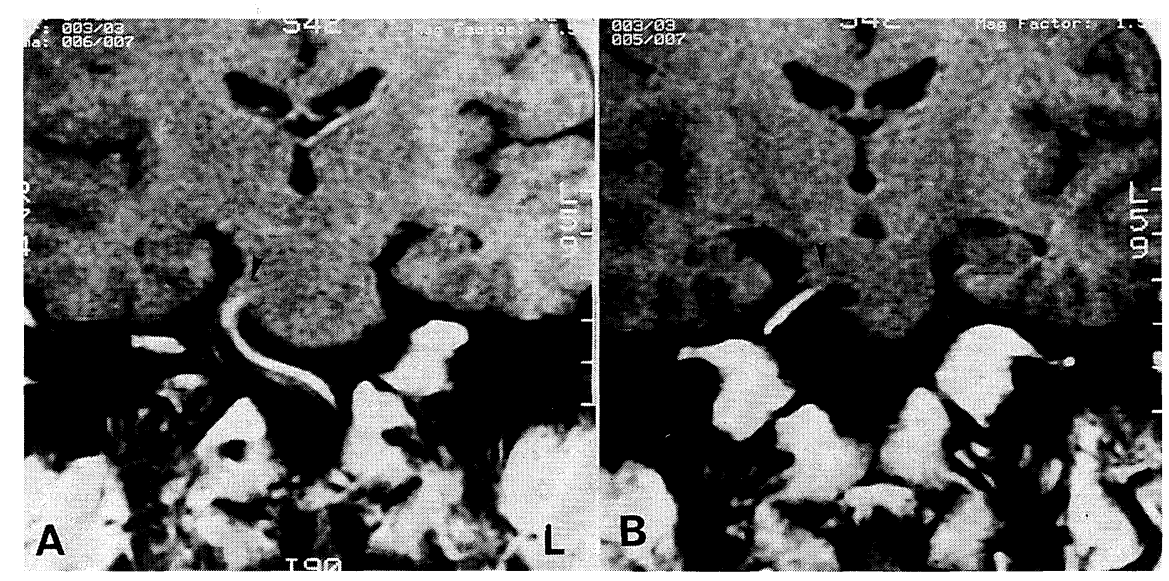

Fig. 1. T1-weighted MRI of coronal section of brainstem. Panel A shows the tortuous basilar artery wrapped around the pontine base to reach the dorsal aspect of the right mid-pons where the artery appears in direct contact with the trigeminal root entry zone (arrow head). Panel B, the section $4 \mathrm{~mm}$ rostral to panel $\mathrm{A}$, demonstrates the lateral pons being deeply indented by the looped basilar artery. The laminar flow of the ectatic artery appears to have given rise to a linear high-low signal intensity (arrow head) that represents the flow void effect. 


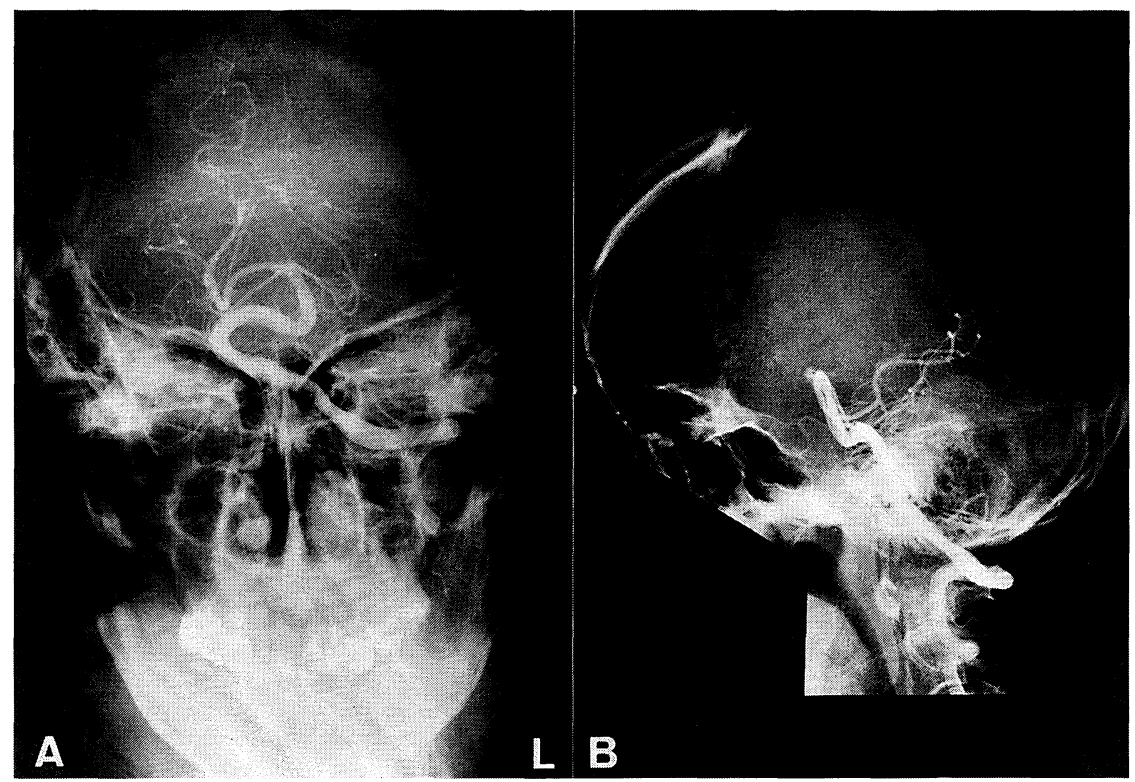

Fig. 2. Cerebral angiogram. Panel A shows that the ectatic basilar artery markedly deviated to the right side. Panel B shows that the bifurcation of artery markedly projected above the clinoid process.

\section{Discussion}

The pathologic conditions responsible for trigeminal neuralgia was as follows: multiple sclerosis, arteriovenous malformation, basilar artery aneurysm, epidermoid, meningioma, acoustic neuroma, trigeminal neuroma, arachnoiditis, compression by adjacent vessels. Multiple sclerosis accounted for about $3 \%$ of patients with trigeminal neuralgia (Penman 1968). The most consistent pathologic lesion in this group of patients is a demyelination plaque in the root entry zone of the fifth nerve in the pons. No case with such a plaque demonstrated by MRI has reported as yet. But Hutchins et al. (1989) reported a case with a focal multiple sclerosis plaque in the vicinity of main sensory nucleus of the fifth nerve wihch was visualized by MRI. Janneta (1981) reported that compression of the trigeminal root entry zone by tumors accounted for 15 cases out of 411 patients with trigeminal neuralgia. It has been shown that MRI is useful for the differential diagnosis and the evaluation of spread of primary trigeminal tumors (Yuh et al. 1988; Saito et al. 1989; Beges at al. 1992).

Vascular compression at the root entry zone of trigeminal nerve in the pons has been documented as a major cause of trigeminal neuralgia (Dandy 1934; Kempe et al. 1969; Janneta 1976, 1977, 1982; Haines et al. 1979). Such vascular compression is mostly caused by a rather small arterial loop of superior cerebellar artery or anterior inferior cerebellar artery and infrequently by a small vein. 
TABLE 1. Trigeminal neuralgia caused by megadolichobasilar anomaly

\begin{tabular}{llllc}
\hline \multicolumn{1}{c}{ Authors } & Year & $\begin{array}{c}\text { Number of } \\
\text { cases }\end{array}$ & $\begin{array}{c}\text { Methods for } \\
\text { diagnosis }\end{array}$ & $\begin{array}{c}\text { Surgical } \\
\text { confirmation }\end{array}$ \\
\hline Dandy & 1934 & $6^{*}$ & & + \\
Boeri and Passerini & 1964 & 1 & CAG $^{\mathrm{a}}$ & - \\
Petty and Southby & 1977 & $1^{*}$ & & + \\
Waga et al. & 1979 & $1^{*}$ & CAG & + \\
Niizuma et al. & 1981 & 1 & CAG, CT & - \\
Yu et al. & 1982 & 1 & CAG & - \\
Resta et al. & 1984 & 7 & CAG & - \\
Takamiya et al. & 1985 & $1^{*}$ & CAG, Double con- & + \\
& & & trast CT & + \\
Lye & 1986 & $4\left(^{*}\right)$ & DSA ${ }^{\mathrm{b}}, \mathrm{CT}$ & + \\
Hutchins & 1989 & $1^{*}$ & MRI & - \\
\hline
\end{tabular}

${ }^{\mathrm{a}} \mathrm{CAG}$, cerebral angiography; ${ }^{\mathrm{b}} \mathrm{DSA}$, intravenous digital subtraction angiography.

${ }^{*}$ Cases whose trigeminal nerves were directly compressed by the main trunk of ectatic vertebrobasilar system.

Cases with trigeminal neuralgia associated with megadolichobasilar anomaly have been rarely documented (Table 1). In addition, cases with neuroimaging or surgical evidence of the nerve compression by the main trunk of an ectatic basilar artery as presented here have been reported more rarely (Dandy 1934; Petty and Southby 1977; Waga et al. 1979; Takamiya et al. 1985; Lye 1986).

Routine angiography was not helpful to reveal the vessel responsible for trigeminal neuralgia (Janneta 1976; Resta et al. 1984). Although Lye emphasized the value of intravenous digital subtraction angiography in the examination of basilar artery ectasia causing trigeminal neuralgia (Lye 1986), it was unable to reveal the site of compression, since the precise relationship of the trigeminal root to adjacent vessels is difficult to be visualized by angiography alone. Takamiya et al. (1985) reported that in a case with both trigeminal neuralgia and hemifacial spasm caused by a tortuous vertebrobasilar system, double-contrast computed tomography using both air and iodinated contrast medium was useful to evaluate the relationship between the involved nerves and the pathogenic vessels prior to surgery. But their method not only requires special skill but also is invasive.

On the other hand, MRI is non-invasive and capable of disclosing the relationship of the involved nerve to the pathogenic vessel. Hutchins et al. (1989) reported a case with trigeminal neuralgia in whom displacement of the fifth nerve by megadolichobasilar artery was visualized by MRI. Recently MR angiography has been used to scrutinize the relationship of the cranial nerves to adjacent vessels. It was reported that MR tomographic angiography (Adler et al. 1992) or 3D-FISP images of MR angiography was useful (Felber et al. 1992) for visualizing the relatinship of the seventh nerve to adjacent vessels in facial spasm. 
As to surgical treatment, gangliolysis, rhizotomy or microvascular decompression is used. Microvascular decompression is the most excellent in relief of pain, morbidity and recurrence rates (Loeser 1989). Gangliolysis is safer and easier, but frequencies of recurrences are larger as compared with the other methods (Loeser 1989). Rhizotomy is as excellent as microvascular depression in both pain relief and recurrences, but morbidity is higher than microvascular decompression. Since an ectatic tortuous basilar artery is sclerotic and immovable, it is difficult to decompress the nerve in the usual manner. Takamiya et al. (1985) used a fenestration clip and decompressed the nerves. Waga et al. (1979) performed rhizotomy at the portio major in place of decompression.

\section{References}

1) Adler, C.H., Zimmerman, R.A., Savino, P.J., Bernardi, B., Bosley, T.M. \& Sergott, R.C. (1992) Hemifacial spasm: evaluation by magnetic resonance imaging and magnetic resonance tomographic angiography. Ann. Neurol., 32, 502-506.

2) Beges, C., Revel, M.P., Gaston, A., Brugieres, P., Meder, J.F. \& Martin, N. (1992) Trigeminal neuromas: assesment of MRI and CT. Neuroradiology, 34, 179-183.

3) Boeri, R. \& Passerini, A. (1964) The megadolichobasilar anomaly. J. Neurol. Sci., 1, 475-484.

4) Dandy, W.E. (1934) Concerning the cause of trigeminal neuralgia. Am. J. Surg., 24, 447-455.

5) Felber, S., Birbamer, G., Aichner, F., Poewe, W. \& Kampfl, A. (1992) Magnetic resonance imaging and angiography in hemifacial spasm. Neuroradiology, 34, 413416.

6) Haines, S.J., Martinez, A.J. \& Janneta, P.J. (1979) Arterial cross compression of the trigeminal nerve at the pons in trigeminal neuralgia. J. Neurosurg., 50, 257-259.

7) Hutchins, L.G., Harnsberger, H.R., Hardin, C.W., Dillon, W.P., Smoker, W.R. \& Osborn, A.G. (1989) The radiologic assessment of trigeminal neuropathy. Am. J. Roentgenol., 153, 1275-1282.

8) Janneta, P.J. (1976) Microsurgical approach to the trigeminal nerve for tic douloureux. Prog. Neurol. Surg., 7, 180-200.

9) Janneta, P.J. (1977) Observations on the etiology of trigeminal neuralgia, hemifacial spasm, acoustic nerve dysfunction and glossopharyngeal neuralgia, definitive microsurgical treatment and results in 117 patients. Neurochimurgia, 20, 145-154.

10) Janneta, P.J. (1981) Vascular decompression in trigeminal neuralgia. In: The Cranial Nerves, edited by M. Samii \& P.J. Janneta, Springer-Verlag, New York, pp. 331-340.

11) Kempe, L.G., Colonel, M.C. \& Smith, D.R. (1969) Trigeminal neuralgia, facial spasm, intermedius and glossopharyngeal neuralgia with persistent carotid basilar anastomosis. J. Neurosurg., 32, 445-451.

12) Loeser, J.D. (1989) Tic douloureux and atypical face pain. In: Textbook of Pain, edited by P.D. Wall \& R. Melzack, Churchill Livingstone, Edinburgh, London, Melbourne and New York, pp. 535-543.

13) Lye, R.H. (1986) Basilar artery ectasia: An unusual case of trigeminal neuralgia. J. Neurol. Neurosurg. Psychiatry, 49, 22-28.

14) Niizuma, H., Ikeda, S. \& Ohyama, H. (1981) Trigeminal neuralgia and hemifacial spasm caused by the compression of tortuous vertebrobasilar system - A case report. No Shinkei Geka, 9, 1167-1170. (in Japanese) 
15) Penman, J. (1968) Trigeminal neuralgia. In: Handbook of clinical neurology, edited by P.J. Vinken \& G.W. Bruyu, North-Holland Publishing Co., Amsterdam, vol. 5, pp. 296-322.

16) Petty, P.G. \& Southby, R. (1977) Vascular compression of lower cranial nerves: Observations using microsurgery, with particular reference to trigeminal neuralgia. Aust. N.Z.J. Surg., 47, 314-320.

17) Resta, M., Gentile, M.A., Cuouzo, F. Di., Vinjau, E., Brindicci, D. \& Carella, A. (1984) Clinical-angiographic correlations in 132 patients with megadolichobasilar anomaly. Neuroradiology, 26, 213-216.

18 Saito, A., Nakazawa, T., Matsuda, M. \& Handa, J. (1989) Comparison of computed tomographic scanning and magnetic resonance imaging in diagnosis of trigeminal schwannoma. Report of four cases. Neurol. Med. Chir. Tokyo, 29, 1101-1106. (in Japanese)

19) Takamiya, Y., Toya, S., Kawase, T., Takenaka, N. \& Shiga, H. (1985) Trigeminal neuralgia and hemifacial spasm caused by a tortuous vertebrobasilar system. Surg. Neurol., 24, 559-562.

20) Waga, S., Morikawa, A. \& Kojima, T. (1979) Trigeminal neuralgia: Compression of the trigeminal nerve by an elongated and dilated basilar artery. Surg. Neurol., 11, $13-19$.

21) Yu, Y. L., Moseley, I.F., Pullicino, P. \& McDonald, W.I. (1982) The clinical picture of ectasia of the intracerebral artery. J. Neurol. Neurosurg. Psychiatry, 45, 29-36.

22) Yuh, W.T., Wright, D.C., Barloon, T.J., Schultz, D.H. \& Sato, Y. \& Cervantes, C.A. (1988) MR imaging of primary tumors of trigeminal nerve and Meckel's cave. $\mathrm{Am}$. J. Roentgenol., 151, 577-582. 